\title{
Velocidad y efecto de la pelota en el momento del impacto en los servicios de tenis: Confiabilidad de un instrumento para medir el movimiento de la pelota (TRACKMAN)
}

\author{
Bumpei Sato, Ryo Wakatsuki, Yu Kashiwagi y Kazuo Funato
}

\begin{abstract}
RESUMEN
Para verificar la confiabilidad de la precisión de las mediciones realizadas con TRACKMAN analizamos 3 tipos de servicio (Plano, Cortado, con Kick), y comparamos los resultados con los valores obtenidos con el sistema de captura del movimiento en 3D (VICON). Se observaron fuertes correlaciones lineales positivas en velocidad, calculadas con TRACKMAN $(x)$ y VICON $(y) ;(r=$ $0.996, p<0.01)$, igual que la cantidad de efecto de la pelota; $(r=0.978, p<0.01)$. Esto sugiere que la velocidad y la cantidad de los valores de efecto de la pelota calculados con TRACKMAN, que permiten proporcionar retroalimentación inmediata, son suficientes para la confiabilidad y serían útiles en situaciones de entrenamiento.
\end{abstract}

\author{
Palabras clave: \\ TRACKMAN, VICON, velocidad, \\ efecto \\ Recibido: 29 Mayo 2017 \\ Aceptado: 10 Agosto 2017 \\ Autor correspondiente: \\ Bumpei Sato. \\ Correo electrónico: \\ bumpei0214@yahoo.co.jp
}

\section{INTRODUCCIÓN}

Takahashi(2007), Cross y Pollard (2009) señalaron que la velocidad del servicio incrementó gradualmente durante una década, entre 1999 y 2009, que el servicio de alta velocidad es una importante ventaja para controlar los juegos y puede, por lo tanto, sustentar el hecho de ganar o perder un partido. Sin embargo, su investigación se centró en la velocidad del servicio y en la cantidad de puntos ganados, sin el análisis de sus características.

Entre 2011 y 2017, el autor devolvió servicios de práctica de Roger Federer, uno de los jugadores mejor clasificados del mundo. Esto le demostró (al autor) que además de su alta velocidad, el saque de Federer tiene características particulares, es decir, un mayor efecto, incluyendo la trayectoria de la pelota desde el impacto hasta que entra en el área de servicio, y un movimiento específico tras el bote.

Kreighbaum y Hunt (1978) sugirieron 5 variables que inciden en la trayectoria de la pelota de beisbol golpeada: su velocidad inicial, la dirección de su eje de rotación, la cantidad de efecto, el ángulo de entrega y la densidad del aire. Los servicios de tenis se pueden categorizar en tipos: plano, cortado y con kick, con diferentes combinaciones de velocidad, efecto y trayectoria de la pelota. Además de la velocidad, la trayectoria y la rotación de la pelota, son dos factores que pueden cambiar su comportamiento tras el bote, pueden confundir las predicciones del adversario, e incidir negativamente sobre su habilidad a la hora de recibir y devolver el servicio. En consecuencia, se considera que estos factores pueden inducir a errores y equivocaciones. Estudios previos demuestran que entre los mejores jugadores del mundo, la cantidad de efecto difiere entre los 1os y 20 servicios (Muramatsu ycols.,2010, 2015. Sin embargo, este análisis se realizó por medio de las imágenes tomadas con cámaras de alta velocidad, por lo tanto, fue imposible obtener retroalimentación inmediata de estos datos.

El desarrollo reciente de TRACKMAN, que proporciona retroalimentación inmediata, ha facilitado la medición exacta del movimiento y de la velocidad de la pelota de golf y de beisbol.

TRACKMAN es un instrumento que aplica el efecto Doppler, un sistema de rastreo de proyectiles de uso militar en base a un radar para hacer mediciones específicas. Para el tenis, el 
desarrollo del "TRACKMAN TENNIS RADAR" en 2003 permitió el cálculo inmediato de la trayectoria, la velocidad inicial, la cantidad de efecto, y el recorrido de la pelota, además de su posición, por ejemplo el punto de impacto.

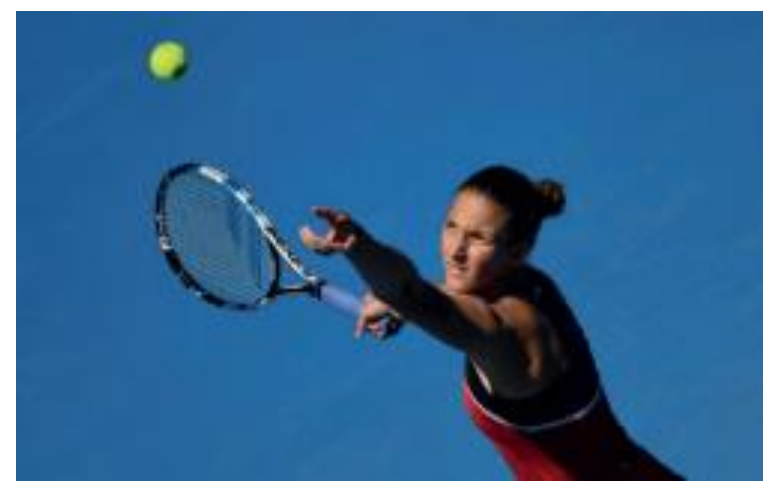

En una investigación anterior, el estudio de la precisión de TRACKMAN se verificó utilizando la cámara de alta velocidad y el arma de velocidad, y dieron como resultado altos niveles de correlación entre la velocidad de la pelota y la cantidad de efecto (Murakami y cols., 2016). Sin embargo, el análisis de los datos de este experimento se realizó visualmente utilizando imágenes obtenidas con una cámara de alta velocidad. En consecuencia, es necesario realizar estudios más detallados 3dimensionales (3D) y analizar el movimiento de la pelota, utilizando múltiples cámaras, para verificar la confiabilidad de TRACKMAN.

En este estudio, medimos simultáneamente la velocidad y la intensidad del efecto de la pelota, los servicios planos, cortados, y con kick, utilizando VICON y TRACKMAN. El análisis del movimiento en 3-D fue llevado a cabo para verificar la confiabilidad de los datos de TRACKMAN.

\section{MÉTODO}

Sujetos: los sujetos involucrados en este estudio incluyeron a un campeón del Campeonato de Tenis de Japón (un jugador profesional), y a 19 alumnos varones de las secciones 1 y 3 de la Federación de Tenis Kanto Inter-Colegiada (media de edad: $23.8 \pm 4.8$ años, altura media: $171.8 \pm 3.3 \mathrm{~cm}$; peso medio: $68.9 \pm$ 4.0 kg; experiencia competitiva media: 11,53 años). Todos los participantes eran diestros. Se explicaron los procedimientos para el experimento y las medidas de seguridad, y se aprobó el consentimiento para todos los sujetos [comité de ética de NSSU \#017H043].

\section{Tarea experimental}

Antes de tomar las mediciones, todos los participantes entraron en calor ejecutando cada uno de los 3 tipos de servicio. Las raquetas utilizadas para el experimento fueron las que usan los participantes regularmente y las pelotas, en todos los casos, fueron Dunlop Fort. Se construyó un sistema local coordinado, colocando 5 marcadores reflectantes en el hemisferio superior de las pelotas.

La tarea consistía en ejecutar cada uno de los 3 tipos de servicio (plano cortado, con kick) a potencia máxima, hasta obtener los datos de 5 pelotas para cada tipo de servicio. Para el plano y con kick, los servicios se golpearon en el centro, (zona T), y para el saque cortado, sobre el costado. Se consideró exitosa la pelota que botaba en la cancha marcada, y dentro del área objetivo $t$. En nuestro estudio, que utilizó el procedimiento experimental para medir velocidades de servicio y cantidad de efecto, se compararon TRACKMAN y VICON (Sakurai y cols.,2012).

\section{Equipamiento experimental}

La velocidad de la pelota y el efecto se midieron utilizando el sistema de captura de movimiento 3-D (VICON MXV5) con 12 cámaras y TRACKMAN. VICON utilizó una computadora personal para controlar las cámaras y realizar las mediciones, cámaras VICON MXV5, una caja de conversión, de 16-bit 64canales AD y una VICON MXV5 para construir las coordenadas 3D. VICON Nexus versión 1.3 fue el programa utilizado para la medición. Se colocaron dos cámaras MXV5 en el techo y 5 cámaras a la derecha y a la izquierda rodeando al sacador. Se calculó la velocidad de la pelota y el efecto para cada servicio en base a las coordenadas de los marcadores reflectivos. Se siguieron las instrucciones del manual de TRACKMAN y se colocó el dispositivo de manera que su centro estuviere en la línea que se extendía desde la marca del centro.
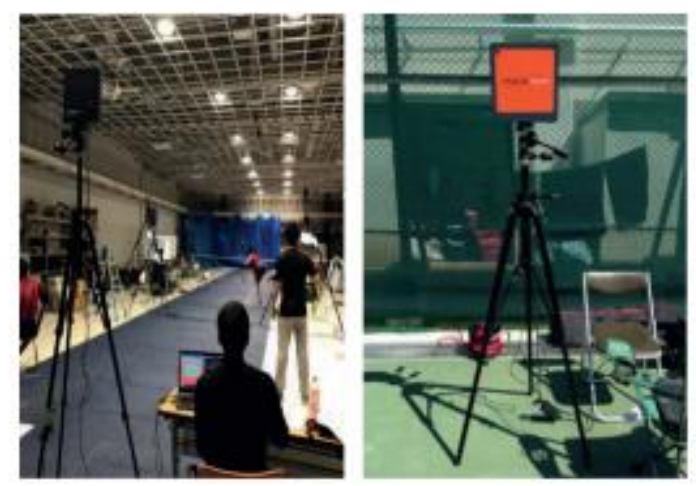

Figura 1. Entorno experimental y TRACKMAN.

Proceso estadístico [definición del sistema de coordenadas]

Para el sistema de coordenadas globales, el eje $\mathrm{X}$ indicaba la dirección de movimiento, el $Z$ la dirección vertical, y el $Y$ perpendicular al eje $X$. Las correlaciones entre la velocidad máxima de la pelota y la cantidad de efecto calculadas con TRACKMAN se determinaron calculando los coeficientes de correlación de producto- momento de Pearson. Para todo el proceso estadístico se utilizó el programa de análisis 
estadístico (IB). Los datos correspondientes a los 3 servicios y a las cifras calculadas con los instrumentos de medición estuvieron sujetos al análisis de variancia de doble vía (ANOVA). Se realizaron múltiples comparaciones con el método Bonferroni cuando se observaron efectos significativos importantes. Una proporción de riesgo de 5\% o menos se consideró significativa estadísticamente.

\section{RESULTADOS Y COMENTARIOS}

\section{Correlación de las velocidades del servicio medidas por} TRACKMAN y VICON

La Figura 2 muestra la correlación entre la velocidad de servicio medida utilizando TRACKMAN (x) y VICON (y). Se observó un alto coeficiente de correlación para los tres servicios; $r=0.996$; $p<0.01)$. También se obtuvieron altos coeficientes de correlación para los tres tipos de servicio (plano: $[r=0.996]$; cortado: $[r=0.992]$; con kick: $[r=0.996], p<0.01)$. En este estudio, se midieron las velocidades de los 3 tipos de servicio ejecutados por los mejores jugadores japoneses utilizando TRACKMAN y la pistola de velocidad. Si bien se obtuvo un alto coeficiente de correlación ( $r=0.997)$ TRACKMAN generalmente tendió a mostrar velocidades mayores (5.5 $\mathrm{km} / \mathrm{h}$ ) que la cámara de alta velocidad (Murakami y cols., 2016). Esto es porque la velocidad no se puede medir con precisión si la superficie de las lentes de la pistola de velocidad (eje óptico) no se alinea con la dirección en la que se mueve el objeto medido, y cuanto mayor es el ángulo entre la onda eléctrica y la trayectoria del objeto, mayor será el error de medición (Morimoto y cols., 2007). En consecuencia, es posible que los valores se vean afectados por el ángulo entre la pistola de velocidad y la trayectoria de la pelota (Murakami y cols. ,2016). Por otro lado, los valores de la velocidad de la pelota obtenidos con TRACKMAN y VICON en este estudio fueron casi exactos. Creemos que se debe a que toda la cancha de individuales podría estar expuesta al radar con TRACKMAN, permitiendo mediciones de velocidad más precisas, en un área más grande que lo que es posible con una pistola de velocidad.

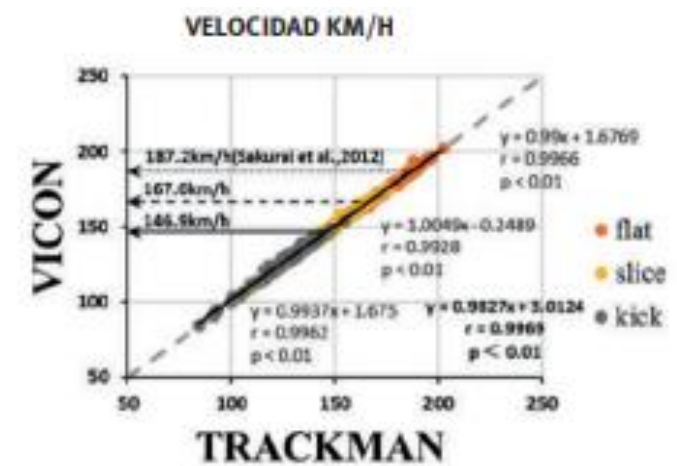

Figura 2. Velocidad de la bola medida por TRACKMAN y VCON.
Cantidad de efecto de la pelota medido por TRACKMAN y VICON

La Figura 3 muestra la correlación del efecto de la pelota (rpm) de tres servicios medidos utilizando TRACKAMAN $(x)$ y VICON (y). Se observó una alta correlación entre los 3 tipos de saque (plano, cortado, con kick; $r=0.978, p<0.01$ ). También se obtuvieron altos coeficientes de correlación para los tres tipos de servicio individualmente (P: $r=0.949] ; \mathrm{E}:[r=0.906,] ; \mathrm{K}:[\mathrm{r}=$ 0.885] $p<0.01$ ). Los valores $r$ para el efecto en los 3 servicios incrementó sucesivamente a medida que el tipo de servicio cambiaba de kick a cortada y a plano. La cantidad de efecto de la pelota se obtuvo con TRACKMAN exponiendo toda la cancha de individuales al radar y basando la información obtenida $300 \mathrm{mts}$ tras el impacto de la pelota. En este estudio, los valores del kick mostraron mayor correlación que los planos o con efecto, cuando se compararon con los valores VICON, aunque el error fue muy leve. Esto se supone que se debe a que este tipo de servicio tiene la mayor cantidad de efecto. De todos modos, la cantidad de efecto calculada con TRACKMAN demostró gran confiabilidad, sugiriendo que este instrumento podría ser útil para las situaciones de entrenamiento.

En cuanto a la velocidad de la pelota, los resultados de doble vía con ANOVA entre TRACKMAN y VICON no demostraron interacción ni diferencias estadísticas significativas. Se observaron efectos significativos entre los tres tipos de servicio (plano, cortado, con kick) pero no hubo diferencias significativas entre los instrumentos de medición.

En cuanto a la cantidad de efecto, los resultados de doble via con ANOVA entre TRACKMAN y VICON no demostraron interacción ni diferencias importantes. Sin embargo, si bien se observaron efectos significativos entre los tres tipos de servicio, no hubo diferencias significativas entre los instrumentos

\section{VELOCIDAD DE GIRO (RPM)}

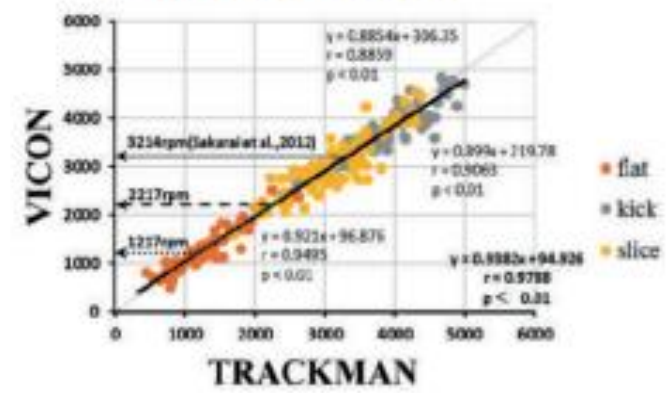

Figura 3. Nivel de giro de la bola medida por TRACKMAN y VICON. 


\section{CONCLUSIÓN}

Para verificar la confiabilidad de la precisión de las mediciones de TRACKMAN, analizamos 3 tipos de servicio, y comparamos los resultados con los valores obtenidos con VICON. Los datos obtenidos mostraron que el coeficiente de correlación de la velocidad de la pelota calculado con TRACKMAN y VICON conforme a $(r=0.996, p<0.01)$, ambos equipamientos de medición no fueron significativos. Además, hubo una alto coeficiente de correlación para la cantidad de efecto de la pelota: $(r=0.978, p<0.01)$ y ambos equipamientos de medición no fueron significativos. Esto sugiere que la velocidad y la cantidad de revoluciones de la pelota calculados con TRACKMAN, que proporcionan retroalimentación inmediata, son muy confiables y serían útiles en situaciones de entrenamiento. Cuando el jugador conoce la información sobre la trayectoria de la pelota inmediatamente, puede hacer los cambios en la cancha con la misma rapidez.

\section{REFERENCIAS}

Cross, R. and Pollard, G. (2009) Grand Slam men's singles tennis 19912009 Serve speeds and other related data. ITF Coaching and Sport Science Review, 49, 8-10.

Kreighbaum, E., \& Hunt, W. A. (1978). Relative factors influencing pitched baseballs. Biomechanics of Sports and Kinanthropometry, 227-236.

Morimoto, Y., Miyanishi, T., Kawaguchi, T(2007). Reliability of ball speed in speed radar gun measurement. : Sendai University bulletin, Vol. 38, No, 2, pp. 10-15.

Murakami, S. Takahashi, H., Muramatsu, T., Sato, B., Sato, M., Koya, N., Kitamura, T., Maeda, A. (2016). Analyzing the speed and spinning frequency of tennis serves: measurements with an instrument that uses radar technology. Research Journal of Sports Performance, 8,361-374.
Muramatsu, T. Takahashi, H., Kaoru, U. (2015). Relationship between speed and spin of tennis serve in world-class tennis players. : Japanese journal of tennis sciences 23, 1-7.

Sakurai, S., Machar, R \& Bruce, E(2012). Ball spin in the tennis serve: Spin rate and axis of rotation, Sports Biomechanics, 12:1, 23-29. https://doi.org/10.1080/14763141.2012.671355

CONTENIDO ITF ACADEMY RECOMENDADO (HAZ CLICK ABAJO)

\section{ITF Academy}

Derechos de Autor (c) 2017 Bumpei Sato, Ryo Wakatsuki, Yu Kashiwagi y Kazuo

$$
\text { Funato }
$$

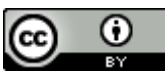

Este texto está protegido por una licencia CreativeCommons 4.0 .

\footnotetext{
Usted es libre para Compartir -copiar y redistribuir el material en cualquier medio o formato- y Adaptar el documento -remezclar, transformar y crear a partir del material- para cualquier propósito, incluso para fines comerciales, siempre que cumpla la condición de:

Atribución: Usted debe dar crédito a la obra original de manera adecuada, proporcionar un enlace a la licencia, e indicar si se han realizado cambios. Puede hacerlo en cualquier forma razonable, pero no de forma tal que sugiera que tiene el apoyo del licenciante o lo recibe por el uso que hace de la obra.

$\underline{\text { Resumendelicencia - Textocompletodelalicencia }}$
} 